\title{
An H I Aperture Synthesis Mosaic Survey of the Large Magellanic Cloud
}

\author{
Sungeun Kim ${ }^{1}$ \\ MSSSO, ANU, Weston Creek PO, Canberra, ACT 2611, Australia
}

L. Staveley-Smith and R. J. Sault

ATNF, CSIRO, P.O. Box 76, Epping, NSW 2121, Australia

M. A. Dopita and K.C. Freeman

MSSSO, ANU, Weston Creek PO, Canberra, ACT 2611, Australia

M. J. Kesteven and D. McConnell

ATNF, CSIRO, P.O. Box 76, Epping, NSW 2121, Australia

We present the results of an $\mathrm{HI}_{\mathrm{I}}$ aperture synthesis mosaic of the Large Magellanic Cloud (LMC), made by combining data from 1344 separate pointing centers using the Australia Telescope Compact Array (ATCA). The resolution of the mosaiced image is $1^{\prime}(15 \mathrm{pc}$, using a distance to the LMC of $50 \mathrm{kpc})$.

The details of this survey and analysis of the results are given in Kim et al. (1998). The $\mathrm{H}$ I map was compared with an $\mathrm{H} \alpha$ image taken with a focal reducing camera and covering the same region of sky as our H I survey (Kim et al. 1999). The $\mathrm{H}$ I disk appears to be remarkably symmetric, and to have a well organized and orderly, if somewhat complex, rotational field (Kim et al. 1998). The mass of the disk component of the LMC is $2.5 \times 10^{9} \mathrm{M}_{\odot}$, and the upper limit to all mass within a radius of $4 \mathrm{kpc}$ is $\sim 3.5 \times 10^{9} M_{\odot}$.

The structure of the neutral atomic ISM in the LMC is dominated by $\mathrm{HI}$ filaments combined with numerous $\mathrm{H}$ I shells and holes (Figure 1). The $\mathrm{H}$ I shell candidates chosen from visual inspection result in the division of the supergiant and giant shell classes on the basis of our estimate of the $\mathrm{H}$ I scale-height in the disk of the LMC (Kim et al. 1999). The expansion velocity increases from about $15 \mathrm{~km} \mathrm{~s}^{-1}$ for the smallest up to about $20-35 \mathrm{~km} \mathrm{~s}^{-1}$ for the giant shells, but the supergiant shells shows no trend with radius (Kim 1998).

We further classify $\mathrm{H}$ I shells into 5 different types, based on the comparision of the $\mathrm{H} \mathrm{I}$ with their associated $\mathrm{H} \alpha$ emission (Kim 1998). In general, there is a weak correlation between the $\mathrm{H}$ I shells and the ionized gas traced out by the $\mathrm{H}$ II regions or $\mathrm{H}$ II shells and a number of the smaller shells have been found on the rim of supergiant shells (Kim et al. 1999).

\footnotetext{
${ }^{1}$ Present Address: Dept. of Astronomy, Univ. of Illinois, Urbana, IL61801, USA
} 


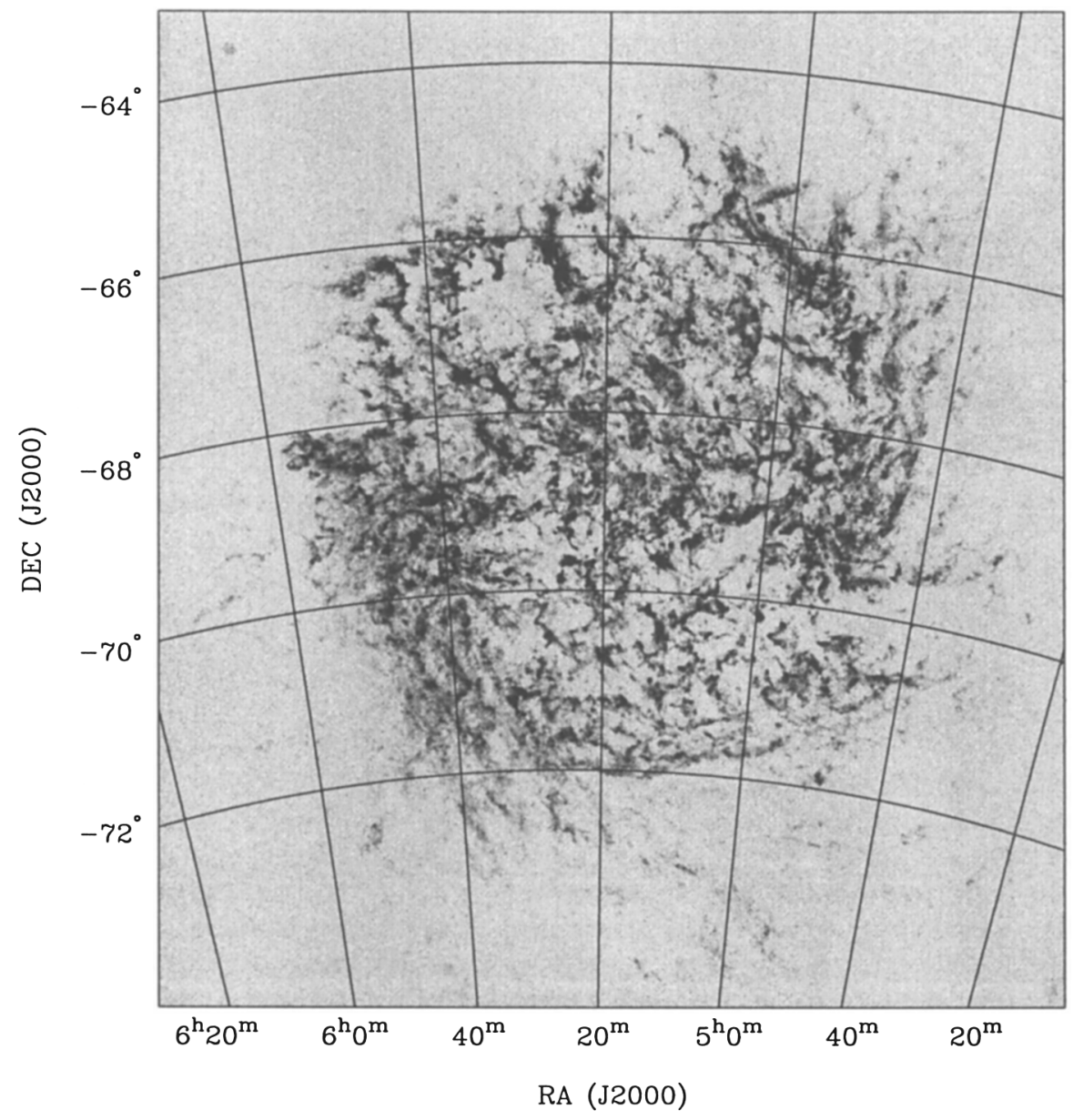

Figure 1. The H I peak brightness temperature map of the LMC.

\section{References}

Kim, S., Staveley-Smith, L., Dopita, M.A., Freeman, K.C., Sault, R.J., Kesteven, M.J., \& McConnell, D. 1998, ApJ, 503, 674.

Kim, S. et al. 1999, in preparation.

Kim, S. 1998, PhD thesis, Australian National University. 